\title{
Energy-momentum tensor for scalar fields coupled to the dilaton in two dimensions
}

\author{
Fernando C. Lombardo* and Francisco D. Mazzitelli ${ }^{\dagger}$ \\ Departamento de Física, Facultad de Ciencias Exactas y Naturales, Universidad de Buenos Aires - Ciudad Universitaria, Pabellón I,
} 1428 Buenos Aires, Argentina

Jorge G. Russo

Theoretical Physics Group, Blackett Laboratory, Imperial College, London SW7 2BZ, United Kingdom

(Received 18 August 1998; published 2 February 1999)

\begin{abstract}
We clarify some issues related to the evaluation of the mean value of the energy-momentum tensor for quantum scalar fields coupled to the dilaton field in two-dimensional gravity. Because of this coupling, the energy-momentum tensor for matter is not conserved and therefore it is not determined by the trace anomaly. We discuss different approximations for the calculation of the energy-momentum tensor and show how to obtain the correct amount of Hawking radiation. We also compute cosmological particle creation and quantum corrections to the Newtonian potential. [S0556-2821(99)05104-8]
\end{abstract}

PACS number(s): 04.60.Kz, 04.62.+v, 04.70.Dy

\section{INTRODUCTION}

In semiclassical and quantum gravity it is of interest to compute the back reaction of quantum fields on the spacetime geometry. Given that a complete four-dimensional calculation is obviously a complicated problem, one may first try to investigate it in two-dimensional models, where nonspherical degrees of freedom are truncated. The twodimensional model of Callan et al. [1] consists of a metric coupled to a dilaton field $\phi$ and conformal matter fields $f$. The action is given by

$$
S=\int d^{2} x \sqrt{g}\left\{\frac{e^{-2 \phi}}{16 \pi}\left[R+4(\partial \phi)^{2}+4 \Lambda^{2}\right]-\frac{1}{2}(\partial f)^{2}\right\} .
$$

By virtue of the conformal symmetry of the classical action, the quantum effects of the matter fields are essentially given by the trace anomaly $\left\langle T_{a}^{a}\right\rangle=R / 24 \pi$. The mean value of the energy-momentum tensor is determined by this anomaly and the conservation law $\left\langle T^{a b}\right\rangle_{; b}=0$. By including $\left\langle T_{a b}\right\rangle$ in the equations of motion, it is possible to study back reaction effects on the spacetime geometry.

In order to make contact with four dimensions, one may consider the usual Einstein-Hilbert action and minimally coupled scalar fields

$$
S=\int d^{4} x \sqrt{g^{(4)}}\left[\frac{1}{16 \pi} R^{(4)}-\frac{1}{2}\left(\partial^{(4)} f\right)^{2}\right] .
$$

For spherically symmetric configurations

$$
\begin{aligned}
d s^{2}= & g_{\mu \nu} d x^{\mu} d x^{\nu}=g_{a b}\left(x^{a}\right) d x^{a} d x^{b} \\
& +e^{-2 \phi\left(x^{a}\right)}\left(d \theta^{2}+\sin ^{2} \theta d \varphi^{2}\right),
\end{aligned}
$$

\footnotetext{
*Electronic address: lombardo@df.uba.ar

${ }^{\dagger}$ Electronic address: fmazzi@df.uba.ar

\#Electronic address: jrusso@ic.ac.uk
}

$$
f=f\left(x^{a}\right), \quad a, b=0,1,
$$

the action reduces to

$$
S=\int d^{2} x \sqrt{g} e^{-2 \phi}\left[\frac{1}{16 \pi}\left(R+2(\partial \phi)^{2}+2 e^{2 \phi}\right)-\frac{1}{2}(\partial f)^{2}\right] .
$$

Unlike model (1), matter fields originating from four dimensions are coupled to the dilaton field.

Similarly, starting with nonminimally coupled scalar fields with the action

$$
S_{\text {matter }}=-\frac{1}{2} \int d^{4} x \sqrt{g^{(4)}}\left[\left(\partial^{(4)} f\right)^{2}+\xi R^{(4)} f^{2}\right],
$$

one gets the following action upon reduction:

$$
\begin{aligned}
S_{\text {matter }}= & -\frac{1}{2} \int d^{2} x \sqrt{g} e^{-2 \phi}\left\{(\partial f)^{2}+\xi f^{2}\right. \\
& \left.\times\left[R^{(2)}+4 \square \phi-6(\partial \phi)^{2}+2 e^{2 \phi}\right]\right\},
\end{aligned}
$$

which, in terms of $\psi=e^{-\phi} f$, reads

$$
S_{\text {matter }}=-\frac{1}{2} \int d^{2} x \sqrt{g}\left[(\partial \psi)^{2}+V \psi^{2}\right]
$$

with

$$
V=\xi R^{(2)}+(4 \xi-1) \square \phi+(1-6 \xi)(\partial \phi)^{2}+2 \xi e^{2 \phi} .
$$

Special cases are $\xi=0$ and $\xi=1 / 6$. For $\xi=1 / 6$, the action is conformal invariant in four dimensions, i.e., invariant under $g_{\mu \nu} \rightarrow e^{2 \sigma(x)} g_{\mu \nu}$ and $f \rightarrow e^{-\sigma(x)} f$. From a two-dimensional viewpoint, this implies [cf. Eq. (8)] $g_{a b} \rightarrow e^{2 \sigma(x)} g_{a b}, \phi$ $\rightarrow \phi-\sigma$, and $\psi \rightarrow \psi$ (or $f \rightarrow e^{-\sigma(x)} f$ ). The matter action in Eq. (4), corresponding to $\xi=0$, is conformal invariant in two dimensions, i.e., under the transformation $g_{a b}$ 
$\rightarrow e^{2 \sigma(x)} g_{a b}, \phi \rightarrow \phi$, and $f \rightarrow f$. For any other $\xi \neq 0,1 / 6$, there is no invariance involving Weyl scalings in the twodimensional model.

Let us now consider the model (4). Due to the conformal symmetry, the trace of the energy-momentum tensor of the scalar fields vanishes classically. There is, however, an anomaly at the quantum level. This anomaly has been computed by a number of authors (see Refs. [2-7]). Some new, interesting effects have been discussed, including the (anti) evaporation of Schwarzschild-de Sitter black holes [8]. However, it has also been claimed (based on an energymomentum tensor obtained by using the conservation law) that quantum effects due to the anomaly produce an ingoing Hawking radiation for Schwarzschild black holes [2,5]. This seems in contradiction with the expectation that the outgoing energy-density flux of Hawking radiation in four dimensions is positive definite, even in the $s$-wave sector. The confusion was partly clarified in a recent paper by Balbinot and Fabbri [9], who pointed out that, due to the coupling between the scalar field and the dilaton, the two-dimensional energymomentum tensor of matter is not conserved and therefore the knowledge of the anomaly is not enough to determine the full energy-momentum tensor. In the same paper they have also raised new puzzles concerning divergences in the mean value of the energy-momentum tensor.

The aim of this paper is to clarify these puzzles and some confusion existing in the literature about the calculation of the energy-momentum tensor of the matter fields in the spherically reduced models. We will compute the effective action and the energy momentum tensor using different approximations, and discuss the validity of each approximation. It will be shown that the energy-momentum tensor can be written as the sum of two terms: an anomalous conserved part and a traceless, non-conserved contribution. As we will see, the last term is relevant for quantum effects on black holes and cosmological spacetimes.

\section{THE EFFECTIVE ACTION}

At the classical level the energy-momentum tensor of the matter fields is given by

$$
T_{a b}=e^{-2 \phi}\left[\partial_{a} f \partial_{b} f-\frac{1}{2} g_{a b}(\partial f)^{2}\right] .
$$

It is important to note that this energy-momentum tensor is traceless and not conserved. Indeed, after using the classical equation of motion for $f$, the divergence is given by

$$
\nabla^{a} T_{a b}=-\frac{1}{2} \partial_{a}\left(e^{-2 \phi}\right)(\partial f)^{2} .
$$

Of course, the quantity that is conserved by Noether theorem in this theory is the complete energy-momentum tensor.

The reason why $T_{a b}$ is not conserved is also clear from the four-dimensional origin of $T_{a b}$. Indeed, from

$$
\nabla^{\mu} T_{\mu \nu}^{(4)}=0
$$

and using Eq. (3), one obtains

$$
\nabla^{a} T_{a b}^{(4)}=2 \partial^{a} \phi T_{a b}^{(4)}-e^{2 \phi}\left(\partial_{b} \phi T_{\theta \theta}^{(4)}+\sin ^{-2} \theta \partial_{b} \phi T_{\varphi \varphi}^{(4)}\right),
$$

which, after using $T_{\mu \nu}^{(4)}=\partial_{\mu} f \partial_{\nu} f-\frac{1}{2} g_{\mu \nu}(\partial f)^{2}$ and $f=f\left(x^{a}\right)$, reproduces Eq. (10).

At the quantum level, the mean value $\left\langle T_{a b}\right\rangle$ is a divergent quantity that must be renormalized. In view of the above discussion, we expect that a covariant renormalization will produce a nonconserved energy-momentum tensor with a trace anomaly. To check this, we must calculate the effective action. The matter action in Eq. (7) can be written as

$$
S_{\psi}=-\frac{1}{2} \int d^{2} x \sqrt{g}\left[(\partial \psi)^{2}+P \psi^{2}\right],
$$

where $P=(\partial \phi)^{2}-\square \phi$.

The Euclidean effective action can be computed using the fact that, at the quantum level, the trace of the energymomentum tensor is given by $T=2 g^{a b}\left(\delta S / \delta g^{a b}\right)$ $=(1 / 24 \pi)(R-6 P)[2,5,7]$. Integrating this equation we obtain

$$
\begin{aligned}
S_{\mathrm{eff}}= & -\frac{1}{8 \pi} \int d^{2} x \sqrt{g} \int d^{2} y \sqrt{g} \\
& \times\left\{\frac{1}{12} R(x) \frac{1}{\square} R(y)-P(x) \frac{1}{\square} R(y)\right\}+S_{\mathrm{eff}}^{\mathrm{I}} \\
\equiv & S_{\mathrm{eff}}^{\mathrm{A}}+S_{\mathrm{eff}}^{\mathrm{I}} .
\end{aligned}
$$

The first term in the above equation $S_{\text {eff }}^{\mathrm{A}}$ produces the expected anomaly, whereas the second term is Weyl invariant and nontrivial due to the coupling between dilaton and scalar fields.

Working in the conformal gauge, the invariant term can be written as

$$
\begin{aligned}
e^{-S_{\mathrm{eff}}^{\mathrm{I}}=} & \operatorname{det}\left[-\square_{f}+P_{f}\right]^{-1 / 2}=\mathcal{N} \int \mathcal{D} \psi e^{-1 / 2 \int d^{2} x \psi\left(-\square_{f}\right) \psi} \\
& \times e^{-1 / 2 \int d^{2} x P_{f} \psi^{2}}
\end{aligned}
$$

where the subindex $f$ indicates that the quantity must be evaluated in a flat metric, and $\mathcal{N}$ is a normalization constant. In some previous works, the invariant term was simply omitted [10]. A possibility is to compute it using an expansion in powers of $P_{f}[11]$ :

$$
\begin{aligned}
S_{\mathrm{eff}}^{\mathrm{I}}= & \int d^{2} x P_{f}(x) D_{1}(x)+\int d^{2} x \int d^{2} y P_{f}(x) D_{2}(x, y) P_{f}(y) \\
& +\cdots
\end{aligned}
$$

Comparing terms of the same order in Eqs. (14) and (15) we obtain $D_{1}(x)=\frac{1}{2} G(x, x), D_{2}(x, y)=\frac{1}{4} G^{2}(x, y)$, where $G$ is the flat Euclidean propagator. Therefore, to second order in the expansion in powers of $P$, the effective action is given by

$$
S_{\mathrm{eff}}^{\mathrm{I}}=\frac{1}{4} \int d^{2} x \int d^{2} y P_{f}(x) G^{2}(x, y) P_{f}(y),
$$


where we have omitted a local divergent term, which can be removed by a counterterm.

The square of the propagator $G^{2}$ was derived in Ref. [12]. It is given by

$$
G^{2}(p)=\frac{1}{2 \pi} \frac{1}{p^{2}} \ln \frac{p^{2}}{\mu^{2}}
$$

Taking this into account, the result up to second order in $P_{f}$ is

$$
\begin{aligned}
S_{\mathrm{eff}}^{\mathrm{I}}= & -\frac{1}{8 \pi} \int d^{2} x \int d^{2} y P_{f}(x) \frac{1}{\square_{f}} \ln \frac{-\square_{f}}{\mu^{2}} P_{f}(y) \\
= & -\frac{1}{8 \pi} \int d^{2} x \sqrt{g} \int d^{2} y \sqrt{g} P(x) \frac{1}{\square} \ln \frac{-\square}{\mu^{2}} P(y) \\
& +\frac{1}{8 \pi} \int d^{2} x \sqrt{g} \int d^{2} z \sqrt{g} \\
& \times \int d^{2} y \sqrt{g} P(x) \frac{1}{\square} R(z) \frac{1}{\square} P(y),
\end{aligned}
$$

where we have performed the Fourier transform of Eq. (17). In the second line we have written the effective action in an explicitly covariant way using that $P_{f}=\sqrt{g} P$ and that the Green function $1 / \square_{f}$ is Weyl invariant. The parameter $\mu$ is an infrared cutoff, and the effective action is $\mu$ dependent because we are computing perturbations around massless fields in two dimensions. Physical results will depend on $\mu$ in this approximation. It is worth noting that this calculation of $S_{\text {eff }}^{\mathrm{I}}$ is valid up to second order in $P$, but no expansion in powers of the curvature $R$ has been performed; in this sense, this derivation differs from the one given in Ref. [11].

To avoid infrared divergences, in Ref. [2] $S_{\text {eff }}^{\mathrm{I}}$ has been computed by assuming that the mass term in Eq. (14) is a constant. This approximation corresponds to neglecting the back scattering of the geometry on the dynamics of the matter fields. In this approximation the effective action reads

$$
\begin{aligned}
S_{\mathrm{eff}}^{\mathrm{I}}= & -\frac{1}{8 \pi} \int d^{2} x P_{f}\left(1-\log \frac{P_{f}}{\mu^{2}}\right) \\
= & -\frac{1}{8 \pi} \int d^{2} x \sqrt{g} P(x)\left(1-\log \frac{P}{\mu^{2}}\right) \\
& -\frac{1}{8 \pi} \int d^{2} x \sqrt{g} \int d^{2} y \sqrt{g} P(x) \frac{1}{\square} R(y) .
\end{aligned}
$$

The last term in Eq. (19) will cancel against a similar term in $S_{\text {eff }}^{\mathrm{A}}$ [see Eq. (13)]. The explicit covariant expression above has been obtained by noting that, in the conformal gauge, $\log (\sqrt{g})=-\square^{-1} R$. As has been shown in Ref. [2], it is possible to go beyond the no-back-scattering approximation by doing perturbations in powers of derivatives of $P$.

In both approximations the effective action can be written as $S_{\text {eff }}=S_{\text {eff }}^{\mathrm{A}}+S_{\text {eff }}^{\mathrm{I}}$. Therefore, a similar decomposition holds for the energy-momentum tensor $\left\langle T_{a b}\right\rangle=\left\langle T_{a b}^{\mathrm{A}}\right\rangle+\left\langle T_{a b}^{\mathrm{I}}\right\rangle$. The anomalous part is independent of the approximation and is given by

$$
\begin{aligned}
\left\langle T_{a b}^{\mathrm{A}}\right\rangle= & \frac{1}{4 \pi} \int d^{2} y \sqrt{g}\left[\nabla_{a} \nabla_{b}-g_{a b} \square\right]_{(x)} P(y) \frac{1}{\square} \\
& -\frac{1}{24 \pi} \int d^{2} y \sqrt{g}\left[\nabla_{a} \nabla_{b}-g_{a b} \square\right]_{(x)} R(y) \frac{1}{\square} \\
& +\frac{1}{8 \pi} \int d^{2} y \sqrt{g}\left[g_{a b} \nabla^{c} \phi \nabla_{c}-2 \nabla_{a} \phi \nabla_{b}+g_{a b}(\partial \phi)^{2}-2 \nabla_{a} \phi \nabla_{b} \phi\right]_{(x)} R(y) \frac{1}{\square} \\
& +\frac{1}{96 \pi} \int d^{2} x \sqrt{g} \int d^{2} y \sqrt{g}\left\{2 \partial_{a} \frac{R(x)}{\square} \partial_{b} \frac{R(y)}{\square}-g_{a b} \partial^{c} \frac{R(x)}{\square} \partial_{c} \frac{R(y)}{\square}\right\} \\
& -\frac{1}{8 \pi} \int d^{2} x \sqrt{g} \int d^{2} y \sqrt{g}\left\{2 \partial_{a} \frac{P(x)}{\square} \partial_{b} \frac{R(y)}{\square}-g_{a b} \partial^{c} \frac{P(x)}{\square} \partial_{c} \frac{R(y)}{\square}\right\} .
\end{aligned}
$$

Note that $\left\langle T_{a b}^{\mathrm{A}}\right\rangle$ has the correct trace anomaly and also contains a traceless, nonconserved part.

In the approximation obtained by expanding in powers of $P$, the nonanomalous part of the energy-momentum tensor reads, up to linear order in $P$, as

$$
\begin{aligned}
\left\langle T_{a b}^{\mathrm{I}}\right\rangle= & -\frac{1}{4 \pi} \int d^{2} y \sqrt{g}\left[g_{a b} \nabla^{c} \phi \nabla_{c}-2 \nabla_{a} \phi \nabla_{b}+g_{a b}(\partial \phi)^{2}-2 \nabla_{a} \phi \nabla_{b} \phi\right]_{(x)} \\
& \times\left(\frac{1}{\square} \ln \frac{-\square}{\mu^{2}} P(y)-\int d^{2} z \frac{1}{\square} R(z) \frac{1}{\square} P(y)\right),
\end{aligned}
$$

while in the no-back-scattering approximation it is given by 


$$
\begin{aligned}
\left\langle T_{a b}^{\mathrm{I}}\right\rangle= & \frac{1}{8 \pi}\left[g_{a b} \nabla^{c} \phi \nabla_{c}-2 \nabla_{a} \phi \nabla_{b}+g_{a b}(\partial \phi)^{2}-2 \nabla_{a} \phi \nabla_{b} \phi\right] \log \frac{P}{\mu^{2}} \\
& -\frac{1}{8 \pi} g_{a b} P-\frac{1}{4} \int d^{2} y \sqrt{g}\left[\nabla_{a} \nabla_{b}-g_{a b} \square\right]_{(x)} P(y) \frac{1}{\square} \\
& -\frac{1}{8 \pi} \int d^{2} y \sqrt{g}\left[g_{a b} \nabla^{c} \phi \nabla_{c}-2 \nabla_{a} \phi \nabla_{b}+g_{a b}(\partial \phi)^{2}-2 \nabla_{a} \phi \nabla_{b} \phi\right]_{(x)} R(y) \frac{1}{\square} \\
& +\frac{1}{8 \pi} \int d^{2} x \sqrt{g} \int d^{2} y \sqrt{g}\left\{2 \partial_{a} \frac{P(x)}{\square} \partial_{b} \frac{R(y)}{\square}-g_{a b} \partial^{c} \frac{P(x)}{\square} \partial_{c} \frac{R(y)}{\square}\right\} .
\end{aligned}
$$

Due to the presence of a term proportional to $\log P,\left\langle T_{a b}^{\mathrm{I}}\right\rangle$ given by Eq. (22) has a singularity when $P \rightarrow 0$. In particular, this seems to imply that $\left\langle T_{a b}^{\mathrm{I}}\right\rangle$ is singular even in Minkowski space, where $P \equiv 0$. To elude this problem, the authors of Ref. [9] proposed a different energy-momentum tensor defined ad hoc. However, we would like to stress that this singularity is an artifact of the no-back-scattering approximation, since the effective action was obtained by assuming that $P$ has a nonzero constant value. In situations where $P \cong 0$, the no-back-scattering approximation breaks down, and it is more appropriate to use the effective action derived by ex- panding in powers of $P$, where no pathology appears at $P$ $=0$. The origin of the $P=0$ singularity in Eq. (22) is quite clear: it is associated with the infrared divergence produced by the two-dimensional field $\psi$ which becomes massless when $P=0$. In the approximation in powers of $P$ (just as in the case of a free scalar field), this is regularized by the infrared cutoff $\mu$.

In both approximations the energy-momentum tensor is not conserved. Indeed, using the expansion in powers of $P$, it is easy to check that the divergence of the energy momentum tensor is nonzero:

$$
\begin{aligned}
\nabla^{b}\left\langle T_{a b}\right\rangle= & \frac{1}{8 \pi}\left[\nabla^{b} \phi \nabla_{a} \nabla_{b}-\nabla^{b} \nabla_{a} \phi \nabla_{b}-2 \nabla_{a} \phi \square-\nabla^{b} \nabla_{a} \phi \nabla_{b} \phi-\nabla_{a} \phi \square \phi\right]_{(x)} \\
& \times \int d^{2} y \sqrt{g}\left\{R(y) \frac{1}{\square}-2 \frac{1}{\square} \ln \frac{-\square}{\mu^{2}} P(y)+2 \int d^{2} z \sqrt{g} \frac{1}{\square} R(z) \frac{1}{\square} P(y)\right\} .
\end{aligned}
$$

On the other hand, in the no-back-scattering approximation we have

$$
\begin{aligned}
\nabla^{b}\left\langle T_{a b}\right\rangle= & -\frac{1}{8 \pi} \nabla_{a} P+\frac{1}{8 \pi}\left[\nabla^{b} \phi \nabla_{a} \nabla_{b}-\nabla^{b} \nabla_{a} \phi \nabla_{b}\right. \\
& \left.-2 \nabla_{a} \phi \square-\nabla^{b} \nabla_{a} \phi \nabla_{b} \phi-\nabla_{a} \phi \square \phi\right] \log \frac{P}{\mu^{2}}
\end{aligned}
$$

As in the classical case Eq. (10), the stress tensor is not conserved when the dilaton field is not constant.

It will be shown below that if the energy-momentum tensor is computed by neglecting the invariant part of the effective action, so that $\left\langle T_{a b}\right\rangle=\left\langle T_{a b}^{\mathrm{A}}\right\rangle$, one obtains wrong results for quantum effects in black hole and cosmological metrics. The same happens if $\left\langle T_{a b}\right\rangle$ is determined from the trace anomaly by imposing a conservation law.

\section{HAWKING RADIATION}

The Hawking radiation for a Schwarzschild black hole formed by gravitational collapse starting from the vacuum has been computed in Ref. [13] and recently discussed in Ref. [9]. The calculation can be easily extended to more general (asymptotically flat) backgrounds. Let us consider the case of a general black hole, formed by gravitational collapse of a shock wave at $v=v_{0}$. For $v<v_{0}$, the geometry is given by the Minkowski metric, i.e.,

$$
d s_{\text {in }}^{2}=-d u_{\text {in }} d v_{\text {in }}, \quad u_{\text {in }}=t-r, \quad v_{\text {in }}=t+r .
$$

For $v>v_{0}$, the geometry is

$$
\begin{gathered}
d s^{2}=-\lambda(r) d u d v \\
u=t-r^{*}, \quad v=t+r^{*}, \quad \frac{d r}{d r^{*}}=\lambda(r),
\end{gathered}
$$

where $\lambda(r)$ vanishes at the event horizon $r=r_{+}$. For example, for a Reissner-Nordström black hole $\lambda(r)=1$ $-2 M / r+q^{2} / r^{2}$. The relation between in and out coordinates follows by matching the geometries at the infalling line $v$ $=v_{0}$ : 


$$
v=v_{\text {in }}, \quad \frac{d u_{\text {in }}}{d u}=\lambda\left(\frac{1}{2}\left(v_{0}-u_{\text {in }}\right)\right) .
$$

Let us first assume that $P(r)=(1 / r) \lambda^{\prime}(r)$ is different from zero everywhere outside the event horizon $r>r_{+}$(this is the case for non extremal black holes). We can therefore use the no-back-scattering approximation. Adding Eqs. (13) and (19) the complete effective action reads

$$
S_{\mathrm{eff}}=-\frac{1}{96 \pi} \int d^{2} x \sqrt{g} R \frac{1}{\square} R+\text { local terms, }
$$

i.e., up to local terms this effective action coincides with the ones for uncoupled scalar fields. In the calculation of Hawking radiation, only nonlocal terms in the effective action are relevant.

The four-dimensional energy-momentum tensor is given by

$$
\begin{aligned}
\left\langle T_{a b}^{(4)}\right\rangle & =\frac{1}{2 \pi} e^{2 \phi} \frac{1}{\sqrt{g}} \frac{\delta S_{\mathrm{eff}}}{\delta g^{a b}}, \\
\left\langle T_{i j}^{(4)}\right\rangle & =\frac{1}{8 \pi} e^{2 \phi} \frac{1}{\sqrt{g}} \frac{\delta S_{\mathrm{eff}}}{\delta \phi} g_{i j},
\end{aligned}
$$

where the $i$ and $j$ indices denote the angular coordinates. The information about Hawking radiation is contained in the components $\left\langle T_{a b}^{(4)}\right\rangle$, which are in turn determined by the twodimensional energy-momentum tensor $\left\langle T_{a b}\right\rangle$. From Eq. (28), and dropping the variation of the local terms, we have

$$
\begin{aligned}
\left\langle T_{a b}\right\rangle= & -\frac{1}{24 \pi} \int d^{2} y \sqrt{g}\left[\nabla_{a} \nabla_{b}-g_{a b} \square\right]_{(x)} R(y) \frac{1}{\square} \\
& +\frac{1}{96 \pi} \int d^{2} x \sqrt{g} \int d^{2} y \sqrt{g}\left\{-g_{a b} \partial^{c} \frac{R(x)}{\square} \partial_{c} \frac{R(y)}{\square}\right. \\
& \left.+2 \partial_{a} \frac{R(x)}{\square} \partial_{b} \frac{R(y)}{\square}\right\}
\end{aligned}
$$

and only the last term contributes to the Hawking radiation [13]. The formal expression $(1 / \square) R$ in the equation above denotes the retarded propagator $G_{\text {ret }}$ acting on the Ricci scalar.

In the conformal gauge $d s^{2}=-e^{2 \rho} d x^{+} d x^{-}$we have $-2 \square \rho=R$, therefore $\rho$ is formally given by $-2 \rho$ $=(1 / \square) R$. The retarded propagator gives $-2 \rho_{\text {in }}=G_{\text {ret }} R$ where $\rho_{\text {in }}$ is one half the logarithm of the scale factor in the in coordinates. The relation between the in and out scale factors is

$$
e^{2 \rho_{\mathrm{in}}}=e^{2 \rho_{\mathrm{out}}} \frac{d u}{d u_{\mathrm{in}}} \frac{d v}{d v_{\mathrm{in}}}=e^{2 \rho_{\mathrm{out}}} \frac{d u}{d u_{\mathrm{in}}} .
$$

The energy flux through $I^{+}$is given by

$$
\left\langle T_{u u}\right\rangle_{I^{+}}=-\frac{1}{12 \pi}\left[\frac{\partial^{2} \rho_{\text {in }}}{\partial u^{2}}-\left(\frac{\partial \rho_{\text {in }}}{\partial u}\right)^{2}\right]_{I^{+}} .
$$

Using Eqs. (31) and (27) we obtain

$$
2\left[\rho_{\text {in }}\right]_{I^{+}}=\log \frac{d u}{d u_{\text {in }}}+\text { const }=-\log \left[\lambda\left(\frac{1}{2}\left(v_{0}-u_{\text {in }}\right)\right)\right]+\text { const. }
$$

Combining the above equations we obtain

$$
\left\langle T_{u u}\right\rangle_{I^{+}}=\frac{1}{192 \pi} \lambda^{\prime 2}
$$

where $\lambda^{\prime}=\lambda^{\prime}\left(r_{+}\right)$. This flux corresponds to a temperature $\left[\left\langle T_{u u}\right\rangle=(\pi / 12) T_{H}^{2}\right]$

$$
T_{H}=\frac{1}{4 \pi} \lambda^{\prime}\left(r_{+}\right)
$$

Note that the above derivation applies for any asymptotically flat black hole with metric $d s^{2}=-\lambda(r) d t^{2}$ $+\lambda^{-1}(r) d r^{2}$. Indeed, the Hawking temperature for a generic black hole of this form (as obtained by going to Euclidean space and compactifying the time direction) is given by $T_{H}=(1 / 4 \pi) \lambda^{\prime}\left(r_{\text {hor }}\right)$, in agreement with the flux (33) obtained above.

The lesson from this calculation is that, as long as $P$ is different from zero, we can apply the no-back-scattering approximation in order to compute Hawking radiation. The main contribution comes from the Polyakov term in the effective action and the result for the Hawking temperature agrees with the well known four-dimensional expression. The next to leading order contribution can be computed as described in Sec. V of Ref. [2].

Let us now consider a background geometry such that $P$ vanishes at the horizon, as is the case for the ReissnerNordström black holes in the extremal limit. In this situation the no-back-scattering approximation still gives the correct result for the Hawking radiation. Moreover, although $P$ vanishes at the horizon, it is easy to check from Eq. (22) that there is no divergence in the energy-momentum tensor. Alternatively, one can compute the Hawking radiation for extremal black holes using the expansion in powers of $P$. Near the horizon the leading contribution in Eq. (13) is given by the nonlocal Polyakov term. Therefore the Hawking temperature is, to leading order in $P$, again given by Eq. (34).

It is important to stress that the expansion in powers of $P$ is not useful to compute the Hawking radiation for Schwarzschild black holes. Indeed, for this geometry $P$ and $R$ are of the same order of magnitude, and one should add the contribution of an infinite number of nonlocal terms in order to obtain the correct radiation.

As a final remark, we stress that if the Weyl invariant part $S_{\text {eff }}^{\mathrm{I}}$ is neglected, the relevant terms for the Hawking radiation are [see Eq. (20)]

$$
\begin{aligned}
& \frac{1}{48 \pi} \int d^{2} x \sqrt{g} \int d^{2} y \sqrt{g} \\
& \quad \times\left\{\partial_{a} \frac{R(x)}{\square} \partial_{b} \frac{R(y)}{\square}-12 \partial_{a} \frac{P(x)}{\square} \partial_{b} \frac{R(y)}{\square}\right\} .
\end{aligned}
$$


Since $P=R / 2$ for the Schwarzschild collapsing geometry, the term proportional to $P$ produces an infalling flux that exceeds by a factor 6 the outgoing one. Thus, if $S_{\text {eff }}^{\mathrm{I}}$ is not taken into account, one would incorrectly obtain a negative energy-density flux of Hawking radiation. This problem appeared in Refs. [2,5].

\section{QUANTUM CORRECTION TO THE NEWTONIAN POTENTIAL}

The different approximations can be tested by computing another observable: the quantum corrections to the Newtonian potential [14]. The four-dimensional semiclassical Einstein equations read

$$
\frac{1}{8 \pi}\left(R_{\mu \nu}-\frac{1}{2} g_{\mu \nu} R\right)={ }^{\text {class }} T_{\mu \nu}^{(4)}+\left\langle T_{\mu \nu}^{(4)}\right\rangle,
$$

where class $T_{\mu \nu}^{(4)}$ is the four-dimensional classical contribution of a point particle of mass $M$, class $T_{\mu \nu}^{(4)}=-\delta_{\mu}^{0} \delta_{\nu}^{0} M \delta^{3}(\vec{x})$ and $\left\langle T_{\mu \nu}^{(4)}\right\rangle$ is the energy-momentum tensor for a quantum massless scalar field.

To solve these equations we consider perturbations around the flat spacetime $g_{\mu \nu}=\eta_{\mu \nu}+h_{\mu \nu}$. For our purposes it is enough to solve the equation for the trace of $h_{\mu \nu}$ to find the quantum corrections. In a perturbative expansion, $h$ $=h^{(0)}+h^{(1)}$, with $h^{(0)}=4 M / r$ coming from the classical solution. The equation for $h^{(1)}$ is

$$
\frac{1}{2 \pi} \nabla^{2} h^{(1)}=g^{\mu \nu}\left\langle T_{\mu \nu}^{(4)}\right\rangle .
$$

At large distances the trace of $\left\langle T_{\mu \nu}^{(4)}\right\rangle$ is given by [14]

$$
\left\langle T^{(4)}\right\rangle=-\frac{M}{8 \pi^{2} r^{5}} \equiv \frac{C}{r^{5}} .
$$

The perturbative solution to the semiclassical Einstein equations is therefore

$$
-\frac{h}{4}=-\frac{M}{r}+\frac{M}{12 \pi} \frac{1}{r^{3}}+\cdots
$$

from which it is possible to read the quantum corrections to the Newtonian potential. For a minimally coupled massless four-dimensional (4D) scalar the stress tensor trace is state dependent. Equation (38) corresponds to computing the trace of the stress tensor in the Boulware state. The expression (38) is in agreement with other calculations of quantum corrections to the Newton potential [15]. This term seems to be, however, missing in the treatment of Ref. [16]. In this work a comparison of numerical and analytic results was made only near the horizon. A complete treatment valid at large distances as well must give a trace of the four-dimensional energy-momentum tensor proportional to $M / r^{5}$ as $r \rightarrow \infty$ in order to reproduce the correct answer for the quantum corrected potential [15].

From the analysis above we see that in order to compute the leading quantum corrections it is necessary to evaluate the (four-dimensional) trace of the energy momentum tensor in the Schwarzschild background. It is interesting to compute it now in the reduced model Eq. (4). On general grounds we expect $g^{\mu \nu}\left\langle T_{\mu \nu}^{(4)}\right\rangle=C / r^{5}$ where $C=C(\mu r)$. The sign of $C$ is very important. Indeed, a negative value of $C$ implies that the Newton constant grows with $r$, as suggested by the fact that there is no screening of the gravitational interaction by quantum matter fields.

The no-back-scattering approximation is not adequate to describe the vacuum polarization in the asymptotically flat region. Indeed, from Eq. (22) we see that for the Schwarzschild metric the energy momentum tensor contains terms proportional to $\left(1 / r^{2}\right) \ln \left(M / \mu^{2} r^{3}\right)$ as $r \rightarrow \infty$. These do not vanish (in fact diverge [9]) as $M \rightarrow 0$. Therefore, the fourdimensional trace $\left\langle T^{(4)}\right\rangle=g^{\mu \nu}\left\langle T_{\mu \nu}^{(4)}\right\rangle=g^{a b}\left\langle T_{a b}^{(4)}\right\rangle+g^{i j}\left\langle T_{i j}^{(4)}\right\rangle$ must be computed using the expansion in powers of $P$ for the effective action. In this approximation we must evaluate Eqs. (20) and (21) in the collapsing metric

$$
d s^{2}=\left(1-\frac{2 M}{r}\right)\left(-d t^{2}+d r^{\star 2}\right)+r^{2} d \Omega^{2},
$$

where $d \Omega^{2}$ is the line element of the unit two sphere, and $r^{\star}$ is given by

$$
r^{\star}=r+2 M \ln \left|\frac{r}{2 M}-1\right| .
$$

In this metric $R=4 M / r^{3}$ and $P=R / 2$. The nonlocal functions $R / \square$ and $P / \square \ln \left(-\square / \mu^{2}\right)$ are computed by means of their Fourier transforms [17], and they are given by

$$
\frac{R}{\square}=\frac{2 M}{r} \quad \text { and } \quad \frac{P}{\square} \ln \frac{-\square}{\mu^{2}}=-\frac{2 M}{r} \ln \tilde{\mu} r .
$$

The four-dimensional components of the energymomentum tensor are given by Eqs. (29). Evaluating Eqs. (20), (21), and taking the functional variation with respect to the dilaton field, we obtain the four-dimensional trace, up to linear order in $M$ :

$$
\left\langle T^{(4)}\right\rangle=-\frac{1}{8 \pi^{2}} \frac{M}{r^{5}} \ln \tilde{\mu} r .
$$

As expected, quantum corrections to the Newtonian potential depend on $\mu$. This correction agrees qualitatively with the four-dimensional result (38), i.e., it has the correct sign. However, if the Weyl invariant part of the effective action were neglected, one would obtain

$$
\left\langle T^{(4)}\right\rangle=\frac{1}{48 \pi^{2}} \frac{M}{r^{5}},
$$

which would lead to quantum corrections to the Newtonian potential with the wrong sign. 


\section{COSMOLOGICAL PARTICLE CREATION}

As another example, in this section we consider particle creation in cosmological backgrounds. Let us consider the metric

$$
d s^{2}=a^{2}(t)\left[-d t^{2}+d r^{2}\right]+a^{2}(t) r^{2} d \Omega_{2},
$$

where $a(t)=1+\delta(t)$ with $\delta \ll 1$ and $\delta \rightarrow 0$ in the far past and future. We denote by $t$ the conformal time.

The total number of created particles is given by the imaginary part of the in-out effective action. This effective action can be obtained from the Euclidean effective action replacing the Euclidean propagators by the Feynman ones. As $P \approx \ddot{\delta}$, the approximation in powers of $P$ is adequate in order to evaluate particle creation rate. Up to lowest order in $\delta$, the Euclidean effective action is given by Eqs. (13) and (18), where the propagators are the flat spacetime ones.

In the conformal vacuum the terms present in the anomalous part of the effective action $\left(S_{\text {eff }}^{\mathrm{A}}\right)$ are real and local for cosmological metrics. The invariant part $S_{\text {eff }}^{\mathrm{I}}$ is nonlocal and contains an imaginary term that gives the particle creation.

Performing a Fourier transform of Eq. (18), and replacing $p^{2} \rightarrow p^{2}-i \epsilon$ we obtain

$$
S_{\text {eff }}^{\text {in-out }}=\frac{1}{16 \pi^{2}} \int d^{2} p|\widetilde{P}(p)|^{2} \frac{1}{p^{2}-i \epsilon} \ln \frac{p^{2}-i \epsilon}{\mu^{2}}+\text { local terms. }
$$

Using the fact that

$$
\ln \frac{p^{2}-i \epsilon}{\mu^{2}}=\ln \left|\frac{p^{2}}{\mu^{2}}\right|-i \pi \theta\left(-p^{2}\right)
$$

the total number of created particles is given by

$$
n_{\mathrm{T}}=\operatorname{Im} S_{\text {eff }}^{\text {in-out }}=-\frac{1}{16 \pi} \int d^{2} p|\widetilde{P}(p)|^{2} \frac{\theta\left(-p^{2}\right)}{p^{2}} .
$$

Since $P=P(t), n_{\mathrm{T}}$ takes the form

$$
n_{T}=\operatorname{Im} S_{\text {eff }}^{\text {in-out }}=\frac{1}{16 V \pi} \int d p_{0}\left|\widetilde{P}\left(p_{0}\right)\right|^{2} \frac{1}{p_{0}^{2}},
$$

where $V$ is the spatial volume.

Because the metric is asymptotically flat for $t \rightarrow \pm \infty$, the Fourier transform $\widetilde{P}\left(p_{0}\right)$ vanishes as $p_{0} \rightarrow 0$. As a result, the total number of created particles $n_{T}$ given in Eq. (48) is a finite quantity. Equation (47) represents the precise twodimensional analogue of the general expression for the total number of created particles in four dimensions (in the case of $\xi=0, m=0$, and $\left.C_{a b c d}=0\right)$ given in Ref. [18].

It is important to note that the effective action coming from the no-back-scattering approximation (28) is not ad- equate to evaluate the particle creation rate because the Polyakov term becomes real and local in the conformal vacuum. This would imply vanishing particle creation, in contradiction with the four dimensional result.

\section{FINAL REMARKS}

To summarize, we have shown that the Weyl invariant part of the effective action contains relevant information about quantum effects in black hole geometries. Neither the effective action nor the mean value of the energy momentum tensor can be completely determined by the trace anomaly when the matter fields are coupled to the dilaton. Neglecting this term, or imposing the conservation law for the twodimensional energy-momentum tensor, leads to wrong results for black hole radiation, quantum corrections to the Newtonian potential and cosmological particle creation.

We have discussed two different approximations in order to compute the invariant part of the effective action: the noback-scattering approximation introduced in Ref. [2], and an expansion in powers of $P$. The no-back-scattering approximation assumes a constant, nonzero value of $P$, and can be improved by performing an expansion in powers of derivatives of $P$ around this nonzero value. This was made in Sec. $\mathrm{V}$ of Ref. [2], where a "back scattering" part of the effective action was added to the no-back-scattering part to get the total $s$-channel effective action. One expects the no-backscattering approximation to be valid for $P^{2} \gg \nabla \nabla P$, and therefore it is not applicable for the evaluation of the mean value of the energy-momentum tensor for nearly flat metrics. However, it is adequate in order to determine the Hawking flux of black holes.

On the other hand, the expansion in powers of $P$ is adequate in situations where $P^{2} \ll \nabla \nabla P$, such as nearly degenerate Reissner-Nordström black holes, or to evaluate $\left\langle T_{a b}(r)\right\rangle$ outside a star whose radius $R$ is such that $R$ $>2 M$. Therefore it is useful to compute quantum corrections to the Newtonian potential. It is also useful to compute cosmological particle creation for weak gravitational fields. In this approximation, the results depend on an infrared cutoff that appears because the model contains massless fields in two dimensions.

\section{ACKNOWLEDGMENTS}

We would like to thank R. Bousso and A. Fabbri for useful comments. The work of F.L. and F.M. was supported by Universidad de Buenos Aires, Conicet and Fundación Antorchas. F.D.M. would like to thank the British Council and the hospitality of the theoretical group at Imperial College, where part of this work has been done. The work of J.R. is supported by the European Commission TMR program Grant No. ERBFMBI-CT96-0982.
[1] C. G. Callan, S. B. Giddings, J. A. Harvey, and A. Strominger, Phys. Rev. D 45, R1005 (1992).

[2] V. Mukhanov, A. Wipf, and A. Zelnikov, Phys. Lett. B 332, 283 (1994).
[3] E. Elizalde, S. Naftulin, and S. D. Odintsov, Phys. Rev. D 49, 2852 (1994).

[4] R. Bousso and S. W. Hawking, Phys. Rev. D 56, 7788 (1997).

[5] W. Kummer, H. Liebl, and D. V. Vassilevich, Mod. Phys. Lett. 
A 12, 2683 (1997); Phys. Rev. D 58, 108501 (1998).

[6] S. Nojiri and S. D. Odintsov, Phys. Rev. D 57, 4847 (1998).

[7] J. S. Dowker, Class. Quantum Grav. 15, 1881 (1998).

[8] R. Bousso and S. W. Hawking, Phys. Rev. D 57, 2436 (1998).

[9] R. Balbinot and A. Fabbri, "Hawking radiation by effective two-dimensional theories," gr-qc/9807123.

[10] A. Mikovic and V. Radovanovic, Class. Quantum Grav. 15, 827 (1998).

[11] I. G. Avramidi, Yad. Fiz. 49, 1185 (1989) [Sov. J. Nucl. Phys. 49, 735 (1989)].

[12] H. Balasin, W. Kummer, O. Piguet, and M. Schweda, Phys. Lett. B 287, 138 (1992).

[13] V. Frolov and G.A. Vilkovisky, in Proceedings of the Second
Seminar in Quantum Gravity, edited by M. A. Markov and P. C. West (Plenum, London, 1983).

[14] D. A. R. Dalvit and F. D. Mazzitelli, Phys. Rev. D 50, 1001 (1994); 56, 7779 (1997).

[15] J. F. Donoghue, Phys. Rev. D 50, 3874 (1994); H. Hamber and S. Liu, Phys. Lett. B 357, 51 (1995); I. Muzinich and S. Vokos, Phys. Rev. D 52, 3472 (1995).

[16] P. R. Anderson, W. A. Hiscock, and D. A. Samuel, Phys. Rev. D 51, 4337 (1995).

[17] M. J. Lighthill, Introduction to Fourier Analysis and Generalised Functions (Cambridge University Press, Cambridge, England, 1958).

[18] J. Frieman, Phys. Rev. D 39, 389 (1989). 